EPJ Web of Conferences 70, 00017 (2014)

DOI: 10.1051/epjconf/20147000017

(C) Owned by the authors, published by EDP Sciences, 2014

\title{
Hadronic observables in hydrokinetic picture of $A+A$ collisions at RHIC and LHC
}

\author{
Yuriy Sinyukov ${ }^{1, \mathrm{a}}$ and lurii Karpenko ${ }^{1,2, b}$ \\ ${ }^{1}$ Bogolyubov Institute for Theoretical Physics NAS of Ukraine, 14-b, Metrolohichna str. Kiev, 03680, Ukraine \\ ${ }^{2}$ Frankfurt Institute for Advanced Studies, Ruth-Moufang-Str. 1, 60438 Frankfurt am Main, Germany
}

\begin{abstract}
The simultaneous description of the hadronic yields, pion, kaon and proton spectra, elliptic flows and femtoscopy scales in hydrokinetic model of A+A collisions is presented at different centralities for the top RHIC and LHC energies. The hydrokinetic model is used in its hybrid version that allows one to switch correctly to the UrQMD cascade at the isochronic hypersurface which separates the cascade stage and decaying hydrodynamic one. The results are compared with pure hybrid model where hydrodynamics and hadronic cascade are matching just at the non-space-like hypersurface of chemical freeze-out. The initial conditions are based on both Glauber- and KLN- MonteCarlo simulations and results are compared. It seems that the observables, especially femtoscopy data, prefer the Glauber initial conditions. The modification of the particle number ratios caused, in particular, by the particle annihilations at the afterburn stage is analyzed.
\end{abstract}

\section{Introduction and model description}

In this paper, we apply the hybrid hydrokinetic model [1] for the description of bulk matter dynamics in heavy ion collisions at top RHIC and $2.76 \mathrm{TeV}$ LHC energies. The unique feature of the model is an advanced procedure of connection from hydrodynamic approach for hot and dense matter above chemical freezeout temperature to cascade model applied for rescatterings in the rarefied hadron gas. We start by discussing the ingredients of the model.

In the present analysis we employ the two variants of initial conditions for the hydrodynamic stage of evolution: the Monte-Carlo Glauber (MC-Glauber) and MC-KLN models.

In MC-Glauber (Monte-Carlo Glauber) model, one starts from sampling the individual nucleon positions inside the nuclei according to an average nucleon density distribution. The nuclei are then arranged with a relative impact parameter $b$ and projected onto the transverse plane. The collision criterion for a pair of nucleons in each event is based on the value of nucleon-nucleon cross-section $\sigma_{N N}$ at the corresponding collision energy. The nucleon-nucleon collisions result in deposition of a certain amount of multiplicity (entropy) to different cells in transverse plane. The contributions to multiplicity (entropy) from the "hard" elementary collisions and from the "soft" ones are different, the former are proportional to the number of binary collisions while the later are associated with number of wounded

\footnotetext{
ae-mail: sinyukov@bitp.kiev.ua

be-mail: karpenko@bitp.kiev.ua
}

This is an Open Access article distributed under the terms of the Creative Commons Attribution License 2.0, which permits unrestricted use, distribution, and reproduction in any medium, provided the original work is properly cited. 
nucleons, or participant number [2]. We use GLISSANDO code [3] to calculate both contributions and suppose that the initial entropy profile in the transverse plane at midrapidity is proportional to a linear combination of the density of wounded nucleons and that of binary collisions,

$$
s\left(x_{T}\right)=C\left(\frac{1-\delta}{2} \frac{d N_{\mathrm{w}}}{d^{2} x_{T}}+\delta \frac{d N_{\mathrm{bin}}}{d^{2} x_{T}}\right) .
$$

Different centrality classes are then defined via the corresponding cuts on the number of wounded nucleons. To obtain smooth initial entropy density profiles for single-shot hydrodynamic run, we average the distributions from large enough ensemble of Monte-Carlo simulations.

In the MC-KLN model of initial conditions [4], the initial entropy density comes from the distribution of gluons in the transverse plane, computed in Kharzeev-Levin-Nardi (KLN) approach [5] for each generated configuration of nucleons in colliding nuclei:

$$
s_{0}\left(x_{T}\right)=\left.C \cdot 3.6 \frac{d N_{g}}{\tau_{0} d^{2} x_{T} d \eta_{s}}\right|_{y=\eta_{s}=0}
$$

To calculate initial conditions in this case, the publicly available code mckln-3.43 [6] is used, and centrality selection and averaging is done in the same way as above.

The quark-gluon plasma and hadronic gas are supposed to be in complete local thermal and chemical equilibrium above the chemical freeze-out temperature $T_{c h}=165 \mathrm{MeV}$. This phase of matter evolution is described with the help of the ideal hydrodynamic approximation. The latter is based on 3+1D (in general case) numerical hydrodynamic code, described in [7]. We use the latticeQCDinspired equation of state of quark-gluon phase [8] together with corrections for small, but non-zero baryon chemical potentials $[7,9]$, matched with chemically equilibrated hadron-resonance gas via crossover-type transition. The hadron-resonance gas consists of all $(N=329)$ well-established hadron states made of u,d,s-quarks, including $\sigma$-meson $\left(f_{0}(600)\right.$. Quantitatively, the EoS table used is close to the recent result of hotQCD collaboration $[10,11]$.

Two different approaches to match hadrodynamic and hadronic cascade stages are used. The first one is standard and corresponds to sudden transition from hydrodynamic regime to UrQMD cascade. In this approach, the distribution of $i$-th sort of hadrons at switching hypersurface is expressed through hydrodynamic and thermodynamic variables $u(x), T(x), \mu_{i}(x)$ by the Cooper-Frye formula. This approach we call traditionally as "hybrid model". With this approach, we switch to hadronic cascade (implemented via UrQMD code) already at the chemical freezeout hypersurface.

A more sophisticated hydrokinetic approach accounts for a gradual particle (hadron) liberation from the hydrodynamically expanding medium based on the particle collision rates in the hadronresonance gas. The approach allows one to build the (non-equilibrium) distribution function accounting for the above mentioned effect on any hypersurface $\sigma_{\mathrm{sw}}$ that is situated behind the one corresponding to hadronization process. Also to avoid potential problems at the non-space-like sectors of switching hypresurface (in Cooper-Frye case), in the consistent approach one should use the spacelike hypersurface to switch to hadronic cascade, which is possible to do with hydrokinetics. For present analysis, in the case of hydrokinetic switching we use $\sigma_{\mathrm{sw}}: \tau=\tau_{\mathrm{sw}}=$ const hypersurface, which is completely space-like. $\tau_{s w}$ is defined from the requirement that the isochronic hypersurface touches the chemical freeze-out isotherm at $r=0: T\left(\tau_{s w}, r=0\right)=T_{c h}$. However, other choices of switching space-like hypersurface are also possible.

Once the distribution function on the switching hypersurface is known, we proceed with the standard (for present event generators) method of Monte-Carlo event generation for input to UrQMD. According to pre-calculated mean particle multiplicities and given distribution functions, we generate 
in each event the coordinates and momenta of all particles with $\left|\eta_{s}\right|<2$ and $|y|<2$ intervals, which are wide enough to study particle production at mid-rapidity. The generated set of particles is then used as input for UrQMD code [12], which computes further elastic and inelastic collisions and decays of unstable hadrons.

\section{Results}

We start from the description of the top RHIC Au+Au data in hHKM model, which is shown in Fig. 1 , and discussion of the model parameters.

The normalization constant for the initial entropy density for both MC-Glauber and MC-KLN cases are defined by the all charged particle multiplicities. We also fix the parameters of chemical freezeout: $T_{\mathrm{ch}}=165 \mathrm{MeV}, \mu_{B}=28 \mathrm{MeV}, \mu_{S}=7 \mathrm{MeV}, \mu_{E}=-1 \mathrm{MeV}$, according to the analysis of particle number ratios from STAR in thermal model $[13,14]$, and include contributions from weak decays to proton spectrum to reproduce STAR results. Note that in our hadron table we also include additional resonance states, e.g., $f_{0}(600), f_{0}(980), a_{0}(980)$ and high mass resonances with $m>2 \mathrm{GeV}$, following by the recent compilation from Particle Data Group [15]. $f_{0}(600)$ is observed as broad resonance structure, with very little knowledge about its decay channels and branching ratios. However, these resonances contribute to both final pion and (less) for proton yields [13], thus modifying particle number ratios, in particular, improve (anti)proton yields at RHIC.

Since there are some discrepancies between STAR and PHENIX multiplicities, we choose initial conditions in the model to reproduce the STAR multiplicities, but compare the results with the spectra and HBT radii measured by both collaborations. Since transverse momentum spectra of identified hadrons are measured in a wide $p_{T}$ range only by PHENIX, we multiply the PHENIX proton spectrum by the factor 2 to better compare its form with the STAR points and the hHKM calculations. For 80$92 \%$ central collisions, where no STAR spectra are available, we fit multiplicity to the PHENIX data. From Fig. 1 it is seen that the kaon multiplicity is over-predicted in hHKM for non-central events (if compared to the PHENIX data), which could be a sign of the incomplete equilibration of strangeness in peripheral collisions [16]. However, this effect is not seen when one compares results with the STAR kaon data.

In line with calculations performed in [17], the early starting time for hydro can be used to mimic the initial transverse flow, developed very effectively to the conventional thermalization time $\tau_{\text {th }} \approx 1$ $\mathrm{fm} / \mathrm{c}$ by non-thermal mechanism. The MC-Glauber initial conditions are thus attributed to the starting time $\tau_{0}=0.1 \mathrm{fm} / \mathrm{c}$, together with some initial flow in the form $\eta_{T}=\alpha \cdot r_{T} / R^{2}(\phi)$, where $R(\phi)$ is the radius of the system as a function of azimuthal angle $\phi$. No essential transverse prethermal flow (except small random one) can be developed to $0.1 \mathrm{fm} / \mathrm{c}$, and the only reason to introduce this flow is to provide corrections to the final radial and elliptic flow that are similar to the corrections brought in by the shear viscosity. We set $\alpha=0.45\left(<v_{T}>=0.023 \mathrm{c}\right)$ for the most central collisions (hybrid model case) or $\alpha=0.9$ corresponding to $\left\langle v_{T}\right\rangle=0.046 \mathrm{c}$ (hydrokinetic switching procedure).

In the middle row of Fig. 1 we compare the elliptic flow coefficients in our and "hybrid" models to the STAR [21] and PHENIX [23] results for all charged hadrons, obtained with event plane method.

The results shown are calculated with rather close set of parameters for all centrality classes; only the parameter of initial flow $\alpha$ increases for centralities $60-70 \%$ and upward, that means that the viscosous corrections to ideal hydro evolution of QGP phase become more pronounced for peripheral collisions (the same conclusion was made in the model based on viscous hydrodynamics in [18]).

For all centralities the hydrokinetic switching case leads to slightly bigger final radial flow, that results in larger effective temperature of the kaon and proton spectra. However, the latter case is definitely favored by interferometry data, and particularly $R_{\text {our }} / R_{\text {side }}$ ratio, as seen in Fig. 1 , bottom. 

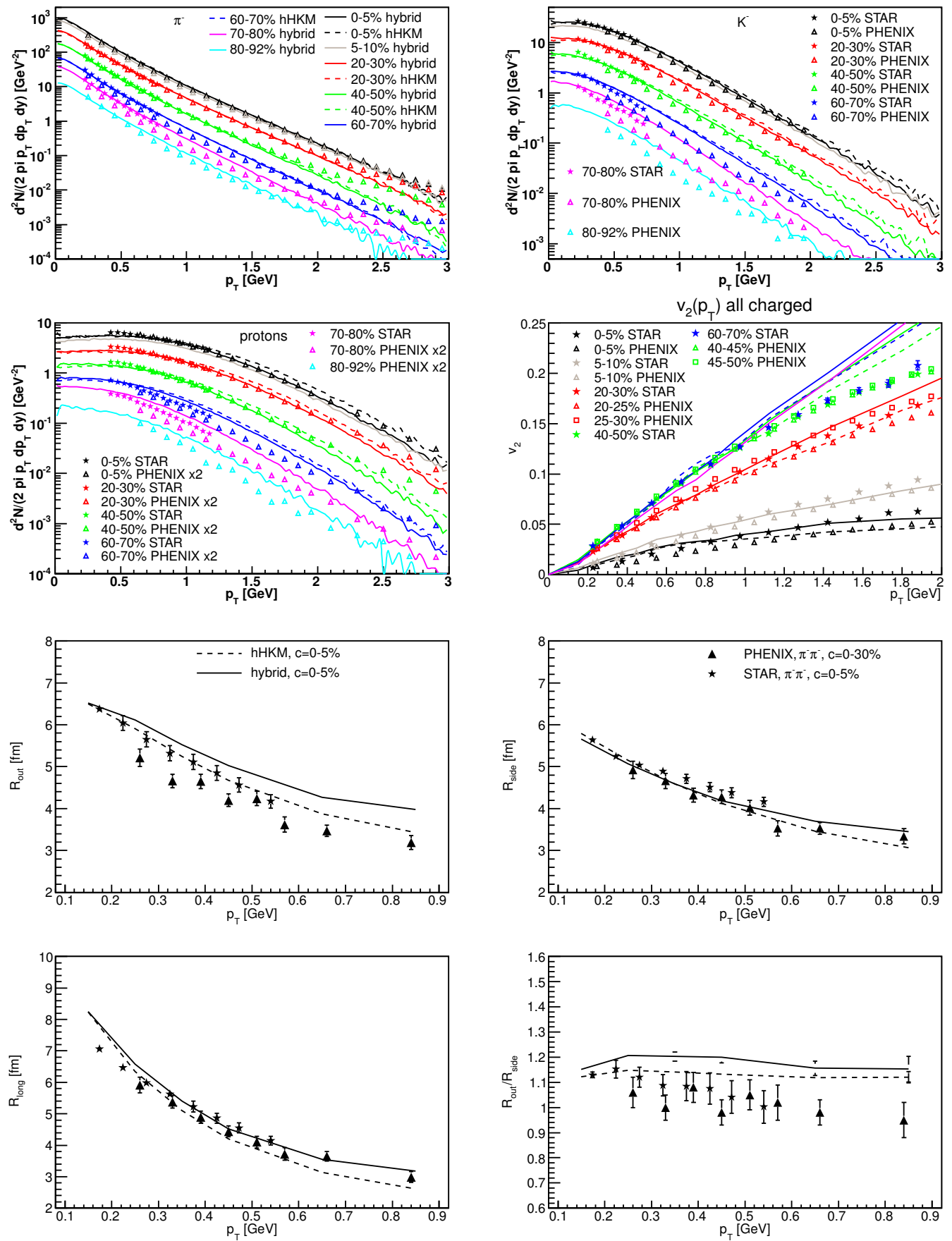

Figure 1. $\pi^{-}, K^{-}$and proton spectra and elliptic flow of all charged hadrons at mid-rapidity for 200A GeV RHIC energy and different centrality classes (top) and HBT-radii of $\pi^{-}$pairs for most central events, calculated in hHKM model and compared to experimental data from STAR [19, 21, 22] and PHENIX [20, 23, 24] collaborations. Dashed lines correspond to hydrokinetic procedure of matching, while solid lines stand for "hybrid" model case. The initial conditions used: $\tau_{0}=0.1 \mathrm{fm} / \mathrm{c}$ and the MC-Glauber profiles for initial entropy density. 

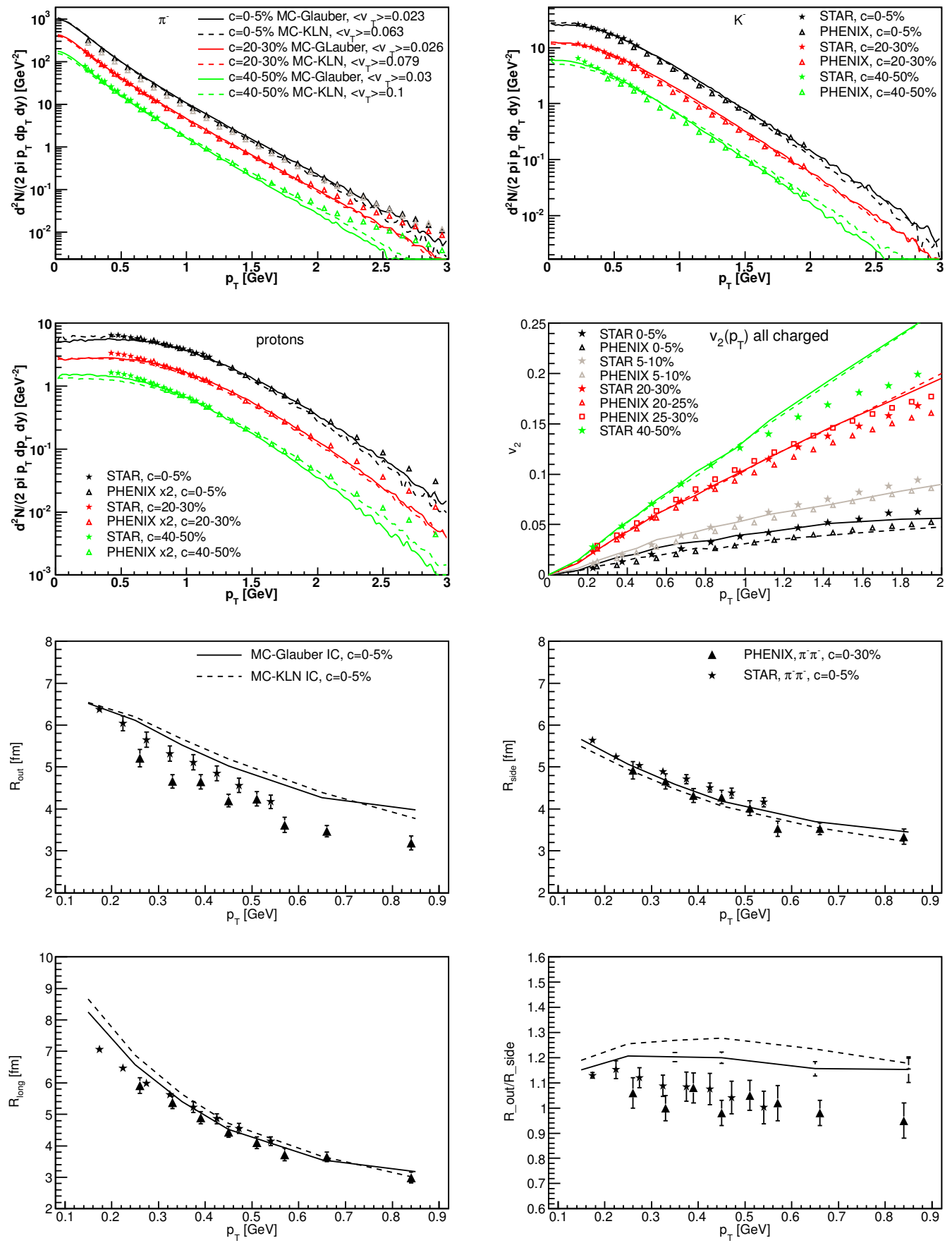

Figure 2. The same as in Fig. 1, calculated for different models of initial conditions for hydrodynamic evolution: MC-Glauber (solid lines) and MC-KLN (dasked lines). Starting times for hydrodynamic evolution are $\tau_{0}=0.1$ $\mathrm{fm} / \mathrm{c}$ for MC-Glauber and $\tau_{0}=0.6 \mathrm{fm} / \mathrm{c}$ for MC-KLN cases. 


\begin{tabular}{|l|l|l|l|l|l|}
\hline & $N_{\pi}$ & $N_{K}$ & $N_{p}$ & $p / \pi$ & $K / \pi$ \\
\hline full & 775 & 123 & 40.5 & 0.052 & 0.158 \\
full- $B \bar{B}$ & 716 & 114 & 50.5 & 0.072 & 0.159 \\
thermal & 679 & 127 & 54 & 0.08 & 0.188 \\
\hline
\end{tabular}

Table 1. Particle multiplicities and particle number ratios, calculated within hHKM model for most central $(0-5 \%) \mathrm{PbPb}$ collisions with $\sqrt{s}=2.76 \mathrm{TeV}$ in differen scenarios of particle production: full scenario (hydro+UrQMD), full- $B \bar{B}$ (baryon-antibaryon annihilator switched off in UrQMD), and thermal model (kinetic phase with resonance decays only).

Next, having fixed the model parameters for MC-Glauber initialization, we study another choice of initial conditions: the MC-KLN model. Similar to the MC-Glauber case, large enough sets of events were generated to obtain smooth initial conditions corresponding to several centrality classes. A change of the shape of initial entropy distribution from MC-Glauber to MC-KLN only, resulted in overestimated values of $v_{2}$ for central and non-central collisions (one can find the same conclusion in [18]). So, finally, for the MC-KLN case a larger value of the initial flow and later hydro start, at $\tau_{0}=0.6 \mathrm{fm} / \mathrm{c}$, is needed, that may correspond to bigger viscous corrections (bigger $\eta / s$ value). The results, compared to MC-Glauber case, are shown in Fig. 2. From Fig. 2 it is also seen that the situation is different for 40-50\% centrality: the same amount of initial flow as in MC-Glauber is enough to suppress $v_{2}$ to its experimental value, however, more strong radial flow is developed. On the contrary, even bigger initial flow for most central events does not help to improve HBT compared to MC-Glauber case. Summarizing the results, MC-Glauber IC model (in particular, the variant with $\delta=0.15$ for the fraction associated with binary scatterings in the initial entropy density) leads to somewhat better than MC-KLN simultaneous description of identified hadron spectra, elliptic flow and interferometry radii for top RHIC energy in hHKM model.

Having fixed model parameter for top RHIC case, we now move to LHC energy to inspect how well the model fits to $2.76 \mathrm{TeV} \mathrm{LHC} \mathrm{Pb}+\mathrm{Pb}$ collisions. First we have to account for the corresponding increase of the initial entropy production at the LHC energy by changing the normalization of the initial energy density profile. As for RHIC, we obtain the normalization $\epsilon_{0}$ for each centrality class from the multiplicity of produced hadrons at mid-rapidity $d N_{\mathrm{ch}} / d \eta$. With bigger initial energy density, hydrodynamic evolution evidently leads to larger effective volume at the chemical freeze-out hypersurface, as well as to bigger transverse flow. The second modification is related to the chemical composition at freeze-out: according to approximate particle-antiparticle symmetry at $\sqrt{s}=2.76 \mathrm{TeV}$ energy, confirmed by ALICE data [25], we set all chemical potentials to zero: $\mu_{B}=\mu_{Q}=\mu_{s}=0$.

Let us start with the particle number ratios obtained in hHKM model for LHC energy. In the simulations we also observe antiparticle/particle symmetry in $\pi^{-} / \pi^{+}, K^{-} / K^{+}, \bar{p} / p$ ratios, which are all close to 1 . From the spectra plots in Fig. 3 one can conclude that (anti)proton multiplicity at midrapidity in hHKM model is only slightly overestimated. Indeed, the nontrivial particle number ratios at mid-rapidity are shown in Table 1 . To understand which factors contribute to successful description of particle number ratios at LHC (and in particular $p / \pi$ ratio), let us calculate hadron yields in different scenarios of evolution at post-chemically equilibrated phase, while keeping the same initial conditions and chemical composition at chemical freeze-out. In this sub-analysis we look at the most central collisions, where rescattering effects in cascade - via UrQMD code - should be most prominent. From Table 1 one can see that both pion and proton yields are minimal for the "thermal model" scenario, where only resonance decays are enabled. Involvement of UrQMD to calculate both elastic and inelastic scatterings (except for baryon-antibaryon annihilation, turned off with CTOption $(19)=1$ ) 
increases somewhat pion yield. Finally, switching $B \bar{B}$-annihilation on suppresses baryon yields, in the same time increases pion yield, thus lowering $p / \pi$ ratio to the value 0.052 , which is quite close to the one measured by ALICE [25]. Thus one can conclude that annihilation processes in UrQMD are essential for successful reproduction of $p / \pi$ ratio at the LHC energy. When going to non-central collisions, $p / \pi$ ratio slightly increases up to 0.058 for $20-30 \%$ centrality (consistently with ALICE data), which should be the result of less amount of inelastic processes due to decreased effective volume at hadronization hypersurface. Since charged hadron density at mid-rapidity for $20-30 \%$ central $\mathrm{PbPb}$ collisions at $2.76 \mathrm{TeV}$ is close to the one for most central collisions at top RHIC energy, one can also conclude that inelastic processes in the cascade play the similar role also at RHIC, modifying particle number ratios and, in particular, decreasing $p / \pi$ ratio. It is worthy noting that the value of the effect depends on the dynamics of the fireball that defines a duration of the afterburn stage and so can differ at RHIC and LHC energies.

In Fig. 3 we show the comparison model/experiment for $2.76 \mathrm{TeV}$ LHC energy. The experimental data are related to elliptic flow coefficients from the 4-particle cumulant method, $v_{2}\{4\}$ [26] and interferometry radii to the most central events measured by the ALICE collaboration [27]. To compare with the experimental anti-proton spectrum from ALICE, we exclude all feed-downs from weak decays, except for $\bar{\Sigma}^{+}$. As a result, we observe some difference compared to ALICE for the mass dependence of the effective temperature (inverse slope) of the transverse momentum spectrum: the resulting anti-proton and kaon spectra are too flat in the model, while the pion spectrum is reproduced much better in a wide $p_{T}$ region. The reason for such a mismatch could be that the imitation of the viscous effects by the initial transverse flow is not so effective at LHC than at RHIC because of a more protracted (viscous) hydrodynamics stage. Concerning interferometry results, in hHKM we get systematically lower $R_{\text {side }}$ and $R_{\text {long }}$ radii than the ALICE data for larger $p_{T}$, however they are within the experimental error bars almost in all $p_{T}$ regions. Note that the rise of interferometry volume observed by the ALICE collaboration is well reproduced in hHKM. Since we keep untouched the model parameters when passing from RHIC to LHC energies, except for a general normalization to the another multiplicities and baryonic chemical potentials at freeze-out, one can conclude that the soft physics at RHIC and LHC is similar.

\section{Conclusions}

A consistent description of the transverse momentum spectra for the most abundant hadrons ( $\pi, K$, $p$ ), elliptic flow coefficients and interferometry radii at the top RHIC $\mathrm{Au}+\mathrm{Au}$ and $2.76 \mathrm{GeV} \mathrm{LHC}$ $\mathrm{Pb}+\mathrm{Pb}$ collisions in the hybrid hydrokinetic model (hHKM) is presented. The different procedures for matching of the hydrodynamic evolution and hadronic cascade - standard Cooper-Frye and hydrokinetic ones, and different models of initial conditions - MC-Glauber and MC-KLN are compared. We show that the hydrokinetic procedure of switching yields better description of the interferometry radii, in particular $R_{\text {out }} / R_{\text {side }}$ ratios. Transition from the top RHIC to LHC collision energy in the model is executed by an increase of initial energy density and fixing exactly chemical potentials at chemical freezeout to be zero. We observe the similarity of the soft physics at the RHIC and LHC energies: the observables are reproduced well in the model also for LHC energy. The contributions of different processes at the hadronic cascade stage to the final hadron yields at LHC are also analyzed. The work was supported by NAS Ukraine (Agreement F4-2013) and SFFR (Agreement F33/24-2013).

\section{References}

[1] Iu. A. Karpenko, Yu. M. Sinyukov, K. Werner, Phys. Rev. C 87(2013) 024914, arXiv:1204.5351 [nucl-th]. 

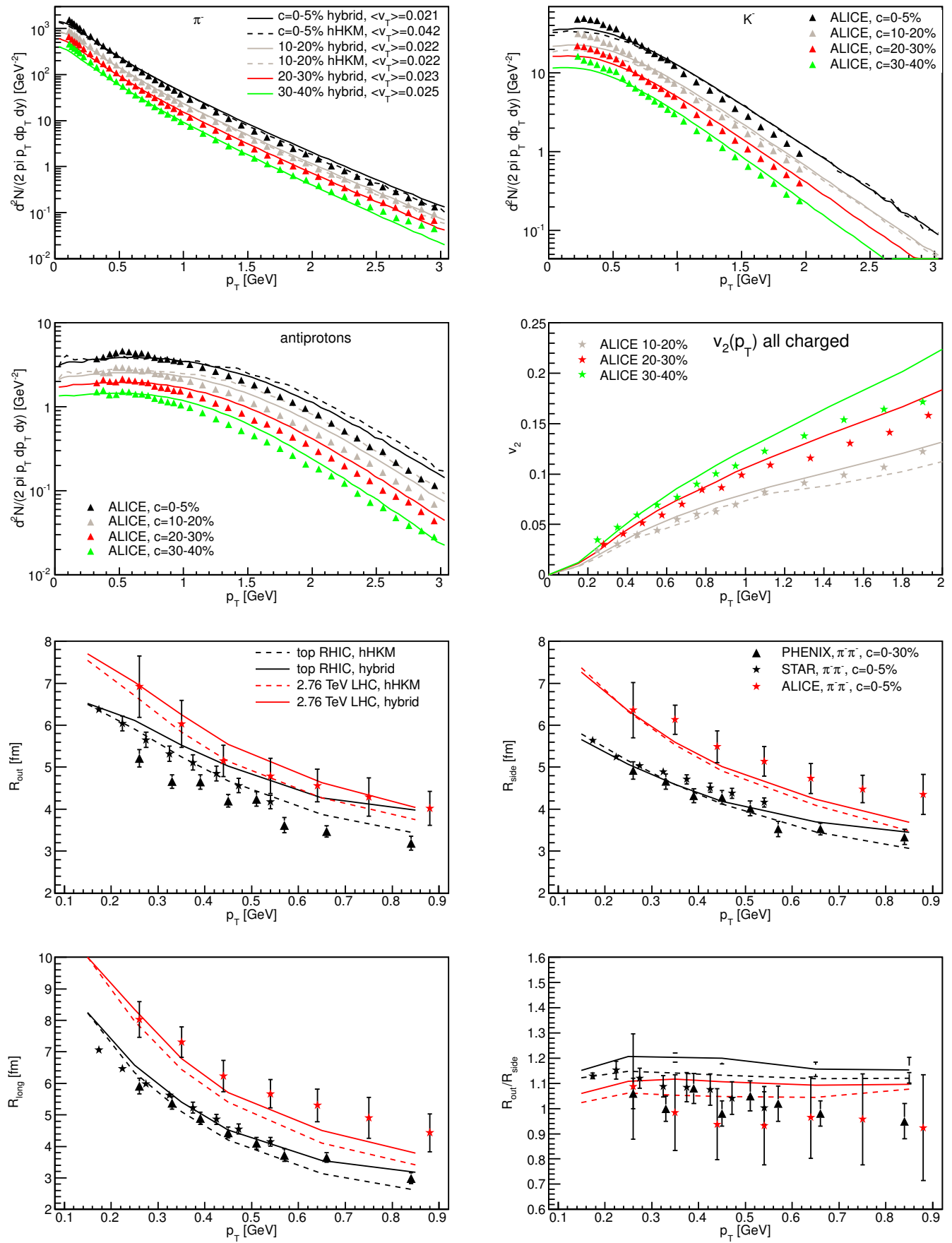

Figure 3. $\pi^{-}, K^{-}$and proton spectra and elliptic flow of all charged hadrons at mid-rapidity for $2.76 \mathrm{TeV}$ LHC energy and different centrality classes (top) and HBT-radii of $\pi^{-}$pairs for most central events, calculated in hHKM model and compared to experimental data from ALICE [25-27]. Dashed lines correspond to hydrokinetic procedure of matching, while solid lines stand for "hybrid" model case. Corresponding HBT-radii for top RHIC energy are shown for comparison purpose. 
[2] D. Kharzeev and M. Nardi, Phys. Lett. B507 (2001) 121.

[3] W. Broniowski, M. Rybczynski, P. Bozek, Comput.Phys.Commun. 180 (2009) 69.

[4] T. Hirano, Y. Nara, Phys.Rev.C79 (2009) 064904.

[5] D. Kharzeev and M. Nardi, Phys. Lett. B 507 (2001) 121; D. Kharzeev and E. Levin, Phys. Lett. B 523 (2001) 79; D. Kharzeev, E. Levin, and M. Nardi, Phys. Rev. C 71 (2005) 054903; D. Kharzeev, E. Levin, and M. Nardi, Nucl. Phys. A 730 (2004) 448.

[6] H.J. Drescher and Y. Nara, Phys. Rev. C76 (2007) 041903.

[7] Iu. A. Karpenko and Yu. M. Sinyukov, Phys. Rev. C 81 (2010) 054903.

[8] M. Laine, Y. Schroder Phys. Rev. D73 (2006) 085009.

[9] F. Karsch, PoS CPOD07 (2007) 026.

[10] M. Cheng et al., Phys. Rev. D 77 (2008) 014511.

[11] A. Bazavov et al., Phys. Rev. D 80(2009) 014504.

[12] S.A. Bass et al., Prog.Part.Nucl.Phys.41 (1998) 255; Prog.Part.Nucl.Phys.41 (1998) 225.

[13] A. Andronic, P. Braun-Munzinger, J. Stachel, Acta Phys.Polon.B 40 (2009) 1005.

[14] J. Manninen, F. Becattini, Phys.Rev.C 78 (2008) 054901; F. Becattini, J. Manninen, J.Phys.G 35 (2008) 104013.

[15] K. Nakamura et al. (Particle Data Group), J. Phys. G 37, 075021 (2010) and 2011 partial update for the 2012 edition.

[16] J. Cleymans, B. Kampfer, M. Kaneta, S. Wheaton, and N. Xu, Phys.Rev. C71 (2005) 054901.

[17] Yu.M. Sinyukov, A.N. Nazarenko, Iu.A. Karpenko, Acta Phys.Polon.B40 (2009) 1109; M. Gyulassy, Iu. Karpenko, A.V. Nazarenko, and Yu.M. Sinyukov, Braz.J.Phys. 37 (2007) 1031.

[18] H. Song, S.A. Bass, U. Heinz, T. Hirano, C. Shen, Phys.Rev. C83 (2011) 054910.

[19] J. Adams, et al (STAR Collaboration), Phys.Rev.Lett. 92 (2004) 112301.

[20] S.S. Adler et al. (PHENIX Collaboration), Phys.Rev.C 69 (2004) 034909.

[21] STAR Collaboration, Phys.Rev. C72 (2005) 014904.

[22] STAR Collaboration, Phys.Rev.C 71 (2005) 044906.

[23] A. Adare et al. (PHENIX Collaboration), Phys.Rev.Lett. 105 (2010) 062301.

[24] S.S. Adler, et al (PHENIX Collaboration), Phys.Rev.Lett 93 (2004) 152302.

[25] R. Preghenella for the ALICE collaboration, Acta Phys. Polon. B43 (2012) 555.

[26] The ALICE Collaboration, Phys.Rev.Lett. 105 (2010) 252302.

[27] The ALICE Collaboration, Phys.Lett.B 696 (2011) 328-337. 